\title{
Single particle properties of $\Lambda$ hypernuclei in the density dependent relativistic hadron field theory
}

\author{
Christoph Keil* and Horst Lenske* \\ ${ }^{*}$ Theoretische Physik Uni Gießen, Heinrich-Buff-Ring 16, 35392 Gießen, Germany
}

\begin{abstract}
The density dependent relativistic hadron field theory is used to describe single particle properties of $\Lambda$ hypernuclei. The discussion focuses on the spin-orbit systematics in the relativistic mean-field formalism by discussing general effects of the $\Lambda$ continuum threshold and the delocalization of the $\Lambda$ wave function. Theoretical predictions for the hypernuclear Auger effect are presented.
\end{abstract}

\section{INTRODUCTION}

Due to the experimental difficulties in persuing hyperonic scattering experiments the modeling of hypernuclei is crucial for investigating interactions in the baryon octet. For this reason the development and application of microscopic models linking in-medium and free interactions is necessary. The extension of the density dependent relativistic hadron field theory (DDRH) [1] into the strangeness sector and to hypernuclei is such a microscopic link. Besides an appropriate microscopic description it is essential to chose suitable systems in which dynamic many-body effects play a minor role in order to get more direct access to generic hyperon interactions. In this respect we present our studies concerned with weakly bound $\Lambda$ states in hypernuclei. It is found that deeply bound $\Lambda$ states are fairly save from subtle dynamical effects - though caution needs to be taken there in other respects. Such deeply bound $\Lambda$ states can ideally be found in heavy hypernuclei. For the investigation of heavy hypernuclei there is, besides the standard spectroscopic methods, also the possibility of the spectroscopy of Auger neutrons [2] as planned in a recently proposed experiment at JLab [3]. We present Auger transition rates for ${ }_{\Lambda}^{209} \mathrm{~Pb}$ calculated within our self-consistent model and discuss the spectroscopic content of the spectra.

\section{THE DDRH MODEL}

The DDRH model [1] is a relativistic Lagrangian field theory with baryonic and mesonic degrees of freedom. In-medium interactions are derived from a Dirac-Brueckner (DB) calculation using the free space Bonn A interaction. The DB self-energies are mapped onto DDRH self-energies leading to vertex functionals that depend on Lorentz scalars of the baryonic field operators. The Lagrange density of DDRH is given by $\mathcal{L}=\mathcal{L}_{B}+\mathcal{L}_{M}+$ $\mathcal{L}_{\text {int }} . \mathcal{L}_{B}$ and $\mathcal{L}_{M}$ denote the free baryonic and mesonic Lagrange densities, whereas the 


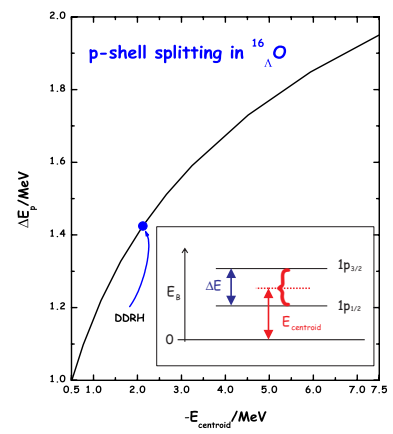

FIGURE 1. The $1 \mathrm{p} \Lambda$ s.o. splitting $\Delta \mathrm{E}$ is plotted versus the centroid energy $\mathrm{E}_{\text {centroid }}$. The insert defines the notation used.

interaction lagrangian is given by:

$$
\begin{aligned}
\mathcal{L}_{\text {int }}= & \bar{\Psi}_{F} \hat{\Gamma}_{\sigma}\left(\bar{\Psi}_{F}, \Psi_{F}\right) \Psi_{F} \sigma-\bar{\Psi}_{F} \hat{\Gamma}_{\omega}\left(\bar{\Psi}_{F}, \Psi_{F}\right) \gamma_{\mu} \Psi_{F} \omega^{\mu} \\
& -\frac{1}{2} \bar{\Psi}_{F} \hat{\vec{\Gamma}}_{\rho}\left(\bar{\Psi}_{F}, \Psi_{F}\right) \gamma_{\mu} \Psi_{F} \vec{\rho}^{\mu}-e \bar{\Psi}_{F} \hat{Q} \gamma_{\mu} \Psi_{F} A^{\mu}
\end{aligned}
$$

Due to the functional structure of the vertices $\Gamma\left(\bar{\Psi}_{F}, \Psi_{F}\right)$ the baryonic equations of motion contain additional rearrangement self-energies which arise due to the variation of $\mathcal{L}_{\text {int }}$ with respect to $\Psi$. These rearrangement self-energies account for static polarization effects in the baryonic medium, assuring the thermodynamical consistency of the theory and Lorentz covariance of the field equations [1].

\section{SINGLE PARTICLE PROPERTIES OF $\Lambda$ HYPERNUCLEI}

\section{Weakly bound $\Lambda$ states}

In this section we point out the subtleties of extracting information on generic hyperon interactions from weakly bound $\Lambda$ systems on two examples: (1) the influence of the close continuum threshold on the structure of $\Lambda$ spin-orbit (s.o.) doublets and (2) the influence of the delocalization of the $\Lambda$ wave function resulting from the weak binding of the $\Lambda$. These effects are independent of the specific interaction and, in general, contribute as an apparent quenching of spin-orbit strengths.

\section{Continuum threshold level compression}

From weakly bound systems, e.g. neutron-rich dripline nuclei [4], it is known that a s.o. doublet which approaches its continuum threshold gets compressed before actually becoming unbound. This is due to the so called avoided level crossing, reflecting the fact that a state which becomes unbound has to cross other states lying lower in the continuum before it reappears as a resonance. In a mean-field description this situation is in particular found in the light p-shell hypernuclei. The effect is investigated 
TABLE 1. Localization coefficients $N_{\Lambda, n}\left(r_{r m s}\right)$ as defined in eq. (2).

\begin{tabular}{c||cc|cc|cc|cc}
\multicolumn{1}{l||}{} & \multicolumn{3}{c}{${ }^{40} \mathrm{Ca}$} & \multicolumn{2}{c}{${ }_{\Lambda}^{51} \mathrm{~V}$} & \multicolumn{2}{c}{${ }^{89} \mathrm{Y}$} & \multicolumn{1}{c}{${ }^{208} \mathrm{~Pb}$} \\
& $N_{\Lambda}$ & $N_{n}$ & $N_{\Lambda}$ & $N_{n}$ & $N_{\Lambda}$ & $N_{n}$ & $N_{\Lambda}$ & $N_{n}$ \\
\hline $1 \mathrm{p}_{3 / 2}$ & 0.51 & 0.71 & 0.59 & 0.75 & 0.68 & 0.81 & 0.79 & 0.88 \\
$1 \mathrm{p}_{1 / 2}$ & 0.50 & 0.71 & 0.60 & 0.76 & 0.69 & 0.82 & 0.80 & 0.86 \\
\hline $1 \mathrm{~d}_{5 / 2}$ & 0.23 & 0.47 & 0.33 & 0.54 & 0.47 & 0.64 & 0.66 & 0.80 \\
$1 \mathrm{~d}_{3 / 2}$ & 0.17 & 0.46 & 0.30 & 0.54 & 0.49 & 0.67 & 0.69 & 0.84 \\
\hline $1 \mathrm{f}_{7 / 2}$ & - & - & - & - & 0.27 & 0.45 & 0.52 & 0.72 \\
$1 \mathrm{f}_{5 / 2}$ & - & - & - & - & 0.26 & 0.47 & 0.55 & 0.77
\end{tabular}

in a model study by varying the $\omega \Lambda \Lambda$ coupling in ${ }_{\Lambda}^{16} \mathrm{O}$ artificially such that the centroid energy $\mathrm{E}_{\text {centroid }}$ of the $1 \mathrm{p}$ s.o. doublet approaches the continuum threshold (for notation see fig. 1). The resulting s.o. splitting $\Delta \mathrm{E}$ depends sensitively on the location of $\mathrm{E}_{\text {centroid }}$ relative to the continuum threshold. Therefore a clean extraction of the generic s.o. interaction from light hypernuclei is hardly to achieve because the threshold compression will always be superimposed on the generic s.o. interaction. However, around the ${ }_{\Lambda}^{40} \mathrm{Ca}$ region the $\Lambda 1 \mathrm{p}$ doublet has moved far enough into the bound region such that the threshold effect ceases to be important.

\section{Wave function delocalization}

Another effect which diminnishes the influence of the s.o. interaction on the observed splitting by an additional reduction is the delocalization of the $\Lambda$ wave function due to the shallow $\Lambda$ potential (e.g. [5]). This comes about because the splitting is proportional to the overlap integral between the single particle density of the considered state and the s.o. potential which is well localized in the nuclear surface. To determine the importance of this delocalization we define the following measure for the fraction of the $\Lambda$ and neutron wave function $F_{\Lambda, n}$ residing inside the nuclear rms radius:

$$
N_{\Lambda, n}(R)=N_{o} \int_{0}^{R} d r r^{2}\left|F_{\Lambda, n}(r)\right|^{2}
$$

where $N_{o}$ is chosen such that $N_{\Lambda, n}(R) \rightarrow 1$ for $R \rightarrow \infty$ and $R=\sqrt{\left\langle r^{2}\right\rangle}$. Results for different nuclei are shown in tab. 1 . It is obvious that this effect is especially pronounced in light hypernuclei and in the weakly bound orbits in the heavy mass region.

We conclude from these investigations, that hyperon interactions still can be determined provided that both the centroid energy and the relative splitting of a doublet are considered. In this respect the new $\gamma$ spectroscopic results, reporting an almost vanishing s.o. splitting in e.g. ${ }_{\Lambda}^{13} \mathrm{C}$ [6], point to an extremely weak effective $\Lambda$ s.o. strength but for decisive conclusions on the generic interaction more strongly bound heavy hypernuclei should be investigated. 




FIGURE 2. The partial life times of the $1 \mathrm{~g} \Lambda$ shell in ${ }_{\Lambda}^{209} \mathrm{~Pb}$ due to Auger neutron emission are plotted versus the kinetic energy of the outgoing neutron. The insert shows the $\Lambda$ and neutron level schemes for this transition where the involved states are marked.

\section{Hypernuclear Auger effect}

Auger rates for the capture of a $\Lambda$ and the de-excitation of heavy hypernuclei are calculated within the DDRH model [1]. Different to a previous attempt [2] our approach treats the binding mean-field, wave functions and interactions in a self-consistent manner. The process is described by the production of a neutron-particle-neutron-hole state due to the de-excitation of the $\Lambda$, where the particle is unbound. A schematical illustration of the process for the $1 \mathrm{~g}$ shell is shown in the insert of fig. 2 . The details of the calculation will be given elsewhere. Fig. 2 shows the partial life times for the $\Lambda$ transitions starting from the $1 \mathrm{~g}$ shell plotted against the kinetic energy of the emitted neutron. The manifestation of the s.o. splitting is marked. Due to the complexity of the full spectrum it will be important to tag the $\Lambda$ initial state in the measurements of Auger spectra. A further analysis of the global spectral features of the hypernuclear Auger effect is in progress.

\section{ACKNOWLEDGMENTS}

This work has been partially supported by DFG grant Le439/4-3 and the European Graduate School "Complex Systems of Hadrons and Nuclei, Gießen-Copenhagen".

\section{REFERENCES}

1. C. M. Keil, F. Hofmann and H. Lenske, Phys. Rev. C 61, 064309 (2000) [nucl-th/9911014].

2. A. Likar, M. Rosina and B. Povh, Z. Phys. A 324, 35 (1986).

3. A. Margaryan et al., JLab letter of intent (2000).

4. G. A. Lalazissis, D. Vretenar, W. Poschl and P. Ring, Nucl. Phys. A 632, 363 (1998) [nuclth/9710013].

5. T. A. Rijken, V. G. Stoks and Y. Yamamoto, Phys. Rev. C 59, 21 (1999) [nucl-th/9807082].

6. S. Ajimura et al., Phys. Rev. Lett 86, 4255 (2001) 